\title{
An asymmetry in the automatic detection of the presence or absence of a frequency modulation within a tone: a mismatch negativity study
}

\author{
Jana Timm $^{1 *}$, Annekathrin Weise ${ }^{1}$, Sabine Grimm ${ }^{2}$ and Erich Schröger ${ }^{1}$ \\ 1 Institut für Psychologie, Universität Leipzig, Leipzig, Germany \\ 2 Institute of Research for Brain, Cognition and Behavior, University of Barcelona, Barcelona, Spain
}

\section{Edited by:}

Andrew J. Oxenham, University of Minnesota, USA

\section{Reviewed by:}

Elyse S. Sussman, Albert Einstein

College of Medicine, USA

Andrew J. Oxenham, University of

Minnesota, USA

*Correspondence:

JanaTimm, Kognitive einschl.

Biologische Psychologie, Institut für

Psychologie, Universität Leipzig,

Seeburgstrße 14-20, 04103 Leipzig,

Germany.

e-mail: jana.timm@uni-leipzig.de
The infrequent occurrence of a transient feature (deviance; e.g., frequency modulation, FM) in one of the regular occurring sinusoidal tones (standards) elicits the deviance related mismatch negativity (MMN) component of the event-related brain potential. Based on a memory-based comparison, MMN reflects the mismatch between the representations of incoming and standard sounds. The present study investigated to what extent the infrequent exclusion of an FM is detected by the MMN system. For that purpose we measured MMN to deviances that either consisted of the exclusion or inclusion of an FM at an early or late position within the sound that was present or absent, respectively, in the standard. According to the information-content hypothesis, deviance detection relies on the difference in informational content of the deviant relative to that of the standard. As this difference between deviants with FM and standards without FM is the same as in the reversed case, comparable MMNs should be elicited to FM inclusions and exclusions. According to the feature-detector hypothesis, however, the deviance detection depends on the increased activation of feature detectors to additional sound features. Thus, rare exclusions of the FM should elicit no or smaller MMN than FM inclusions. In passive listening condition, MMN was obtained only for the early inclusion, but not for the exclusions nor for the late inclusion of an FM. This asymmetry in automatic deviance detection seems to partly reflect the contribution of feature detectors even though it cannot fully account for the missing MMN to late FM inclusions. Importantly, the behavioral deviance detection performance in the active listening condition did not reveal such an asymmetry, suggesting that the intentional detection of the deviants is based on the difference in informational content. On a more general level, the results partly support the "fresh-afferent" account or an extended memory-comparison based account of MMN.

Keywords: event-related brain potentials, mismatch negativity, automatic deviance detection, feature inclusion/exclusion

\section{INTRODUCTION}

On the basis of predictive regularity representations, the human auditory system automatically detects sounds not conforming to the current acoustic context. One function of this system is to bring new, potentially significant information (e.g., a warning signal) occurring outside the focus of attention into consciousness (for reviews, see e.g., Kujala et al., 2007; Näätänen et al., 2007; Winkler et al., 2009). This system has been extensively studied with the mismatch negativity (MMN) component of the event-related brain potential (ERP), firstly described by Näätänen et al. (1978). MMN is elicited by sounds deviating from the currently active regularity representation (deviants), which are presented among a series of sounds confirming the regularity (standards). In the majority of MMN studies, the deviancy affects the whole sound, which features for example the sounds frequency. Here, $M M N$ is elicited relative to the onset of the deviant sound. However, the deviancy can also be confined to a specific temporal part of the sound, which consists, for instance, in a frequency modulation (FM) only lasting for a brief period within the sound. In this case MMN, time-locked to the onset of this modulation, is elicited (e.g., Grimm and Schröger,
2005; Weise et al., 2007). The current study aims at investigating the reverse case, that is, when the standard contains an FM and the deviant is characterized by the absence of an FM.

Up to now it remains unclear to what extent a simple sinusoidal deviant sound, which does not vary in spectral content over time, can be detected by the deviant detection system when occurring among a regular time-variant standard sound. We propose two hypotheses. According to the information-content hypothesis, deviance detection relies on the difference in informational content of the deviant relative to that of the standard (Sinkkonen et al., 1996; Sinkkonen, 1999). As the difference in the informational content does not depend on whether the standard or the deviant contains the FM, the output of the sensory-memory-based comparison mechanism underlying the elicitation of MMN should be the same for both situations. Thus, MMN amplitudes of comparable size should be elicited by rare inclusions or exclusions of an FM in situations in which the standard excludes or includes an FM, respectively. On the other hand, according to the feature-detector hypothesis, we expect an asymmetry in the processing of deviances within sounds as the deviance detection depends on the increased activation of 
feature detectors to additional features which are present in the deviant but absent in the standard (e.g., Bishop et al., 2005). Thus, when the deviant stimulus is characterized by an additional feature that is not present in the standard, MMN amplitude should be enhanced relative to a situation in which the deviant is characterized by an exclusion of a feature that is inherent to the standard sound.

To our knowledge there exists only one study that focused on the processing of deviant sounds that either included or excluded a transient feature (Sabri and Campbell, 2000). Sabri and Campbell (2000) measured MMN to infrequent inclusions or exclusions of clicks of different saliency within complex white noise stimuli. They found MMN occurring during the infrequent inclusion, even when the click within the deviant was of low saliency, whereas they found an MMN-like response for the exclusion only when the click within the standard stimulus was of high saliency. The performance in an active deviance detection task for the low saliency condition was much better for inclusions (hit rate: $p=0.96$ ) than for exclusions (hit rate: $p=0.67$ ), which corresponds to the presence or absence of $\mathrm{MMN}$ in the respective condition. However, the study leaves unanswered whether this was also the case in the high saliency condition. It should be mentioned that the exclusion deviant - although not containing a transient click - still varies in spectral content over time and therefore might be processed differently compared to a pure tone deviant that does not contain any spectro-temporal variation (as also pointed out by Sabri and Campbell, 2000, p. 1507).

There exist a few studies (Winkler and Näätänen, 1993; Nordby et al., 1994; Bishop et al., 2005) reporting a similar pattern of results even though the deviating feature was not transient but affected the whole sound. By using white noise bursts with either including or excluding the 1000-Hz frequency band, Nordby et al. (1994) found a larger MMN for the inclusion (deviant: white noise with $1000-\mathrm{Hz}$; standard: white noise without $1000-\mathrm{Hz}$ ) than for the exclusion (deviant: white noise without 1000-Hz; standard: white noise with 1000-Hz). However, Bishop et al. (2005), who used frequency modulated sounds vs. unmodulated sounds of constant frequency either as standard or deviant, found no MMN when the unmodulated sound was the deviant, even then when participants attended the sounds. Therefore, they concluded that the deviance detection system rather relies on the activity of automatic low-level feature detectors (acting on the basis of stimulus properties) than on high-level feature detectors (acting on the basis of attentiondependent processing).

As they did not report behavioral data with respect to the detection performance of the deviants among the standards, we can only speculate that the missing MMN to the absent modulation within the deviant sound might be due to the fact that the modulation did not reveal sufficient perceptual saliency (e.g., Cusack and Carlyon, 2003). Therefore, in addition to automatic deviance detection, we carefully measured behavioral discriminability of the deviant vs. standard sounds in the present study.

Additionally, we manipulated the time of occurrence of the transient feature within the sound to reveal whether the detection of feature exclusions does (like the detection of feature inclusions) depend on the point in time when the deviance occurs. It is well known that the extent of the processing of a short deviance within a sound depends on the temporal position of the deviance relative to sound onset. This so called temporal distance effect is reflected in decreasing MMN amplitude with increasing temporal distance of the deviance relative to sound onset. When the deviance occurs late within the sound, MMN is attenuated (Näätänen et al., 2004) or even absent (Grimm and Schröger, 2005; Grimm et al., 2006; Weise et al., 2010). MMN latency and reaction times (RTs) during behavioral detection are time-locked to the onset of the deviance within a sound, irrespective of whether the deviance occurs early or late within the sound (Grimm, 2009). Thus, the detection of feature inclusions is triggered by their onset. If a similar pattern is observed for feature exclusions, one can assume that the time point at which the feature occurs in the standard also triggers the detection of its omission in the deviant. This time-precise hypothesis would confirm the "zip-metaphor" of the underlying comparison process, according to which the system compares the incoming stimulus with the memory representation of the predicted sound (derived from the representation of the regular sounds) time point by time point (Grimm and Schröger, 2007). A conceivable alternative, the holistic hypothesis, would suggest that deviants containing the exclusion of a feature are rather detected on the level of a holistic representation of the sound (Warren and Ackroff, 1976; Warren, 1993). In that case sound offset would trigger the final detection process, and RTs (always measured relative to change onset) should thus be longer for early compared to late exclusions.

To examine the information-content and the feature-detector hypothesis with respect to the automatic processing of feature exclusions, we measured MMN to the infrequent exclusion of a brief FM that formed part of the standard tones at different temporal positions (Missing FM early, Missing FM late condition). For control purposes, we measured MMN to the infrequent inclusion of a brief FM at different temporal positions that was absent in the standard tones (FM early, FM late condition). Importantly, the stimuli containing the FM were designed according to a previous study demonstrating that the FM was of sufficient perceptual saliency (Grimm and Schröger, 2005).

According to the information-content hypothesis, we would expect MMN amplitudes of comparable size to occur for both kinds of deviants either including or excluding the transient FM as the relative content of the deviating information is the same. Alternatively, according to the feature-detector hypothesis, we would expect a distinct MMN to the deviant including the transient FM and a reduced or even absent MMN when the deviant excludes the transient FM.

Furthermore, the active condition tests to which extent the detection performance is affected by the feature inclusion vs. exclusion of the transient FM, assuming either a time-precise or a holistic processing. According to the former, for the inclusion and exclusion of the transient FM a parallel pattern in RTs is expected as the detection response is time-locked to the onset of the deviance (FM, Missing FM). In contrast, when the holistic organization of the sound is processed, asymmetries in RTs for the inclusion vs. exclusion of the transient FM should be observable.

\section{MATERIALS AND METHODS PARTICIPANTS}

Thirteen healthy, right-handed volunteers (six male) with selfreported normal hearing, aged 20-30 years (mean age: 23.9 years, SD: 3.1 years), participated in the experiment either for course 
credit or payment. None were taking any medication affecting the central nervous system. Following an explanation of the nature of the experiment, informed consent was obtained from each subject prior to the measurements. The experimental protocol conformed to the Declaration of Helsinki and the ethics guidelines of the German Association of Psychology (ethics board of the Deutsche Gesellschaft für Psychologie, DGPs ${ }^{1}$.

\section{STIMULI AND PROCEDURE}

Three kinds of stimuli were used. Stimuli were $440 \mathrm{~Hz}$ sine wave sounds of $550 \mathrm{~ms}$ duration (including 10-ms rise and 10-ms fall times). Stimuli could contain a short FM of $50 \mathrm{~ms}$ either at 100 or $400 \mathrm{~ms}$ following sound onset. The frequency of these sounds increased for $25 \mathrm{~ms}$ continuously from 440 to $480 \mathrm{~Hz}$ and in the next $25 \mathrm{~ms}$ decreased back to $440 \mathrm{~Hz}$. All sounds were generated with MATLAB ${ }^{2}$. Depending on the respective condition (Missing FM early, Missing FM late, FM early, FM late) the short FM was either a feature of the standard or the deviant sound. In the Missing FM early and Missing FM late conditions, standards featured a transient FM either 100 or $400 \mathrm{~ms}$ after sound onset and deviants were sine wave sounds of constant frequency. In the FM early and FM late conditions, stimuli relations were reversed. Now standards consisted of sine wave sounds with constant frequency and deviants featured a transient FM either 100 or $400 \mathrm{~ms}$ following sound onset. Data were collected using an auditory oddball paradigm with frequently presented standard sounds $(p=0.9)$ and rarely presented deviant sounds $(p=0.1)$. For all four conditions, stimulus onset asynchrony (SOA) was $1100 \mathrm{~ms}$.

During electroencephalographic (EEG) recordings, subjects were seated in a sound-attenuated and electrically shielded chamber watching a self-selected, silent, and subtitled movie while ignoring the auditory stimuli. Auditory stimulation was run via MATLAB (see text footnote 2) using the Cogent2000 toolbox ${ }^{3}$. Stimuli were presented binaurally through headphones with an intensity of about $45 \mathrm{~dB}$ sensation level (adjusted to the individual hearing threshold as obtained with the method described by Kaernbach, 1990). Standard and deviant sounds were presented pseudo-randomized with the restriction that deviant sounds were preceded by at least two standard sounds. Each condition (Missing FM early, Missing FM late, FM early, FM late) was presented in six blocks. Each block consisted of 200 stimuli. Thus, the EEG experiment consisted of 24 experimental blocks, whose order was randomized. A total of 120 deviants and 960 were analyzed for each condition (standards after deviants were rejected from further analysis). In the analysis, the ERP of the deviant sound was compared to the ERP of the physical identical control sound of the reversed condition (e.g., comparison of ERP to deviant sound of condition Missing FM early to ERP physical identical standard sounds of condition FM early).

The passive deviance detection experiment was followed by an active one without any EEG or electrooculographic (EOG) recording. If not otherwise reported stimuli and design were kept identical to those used in the passive EEG experiment. Subjects were instructed to attend to the auditory stimulation while continuously

${ }^{1}$ http://www.dgps.de/dgps/aufgaben/ethikrl2004.pdf

${ }^{2}$ http://www.mathworks.com

${ }^{3}$ http://www.vislab.ucl.ac.uk/cogent_2000.php fixating a cross on the screen. They were instructed to press the left mouse button with their right index finger whenever they detected a deviant sound. The behavioral experiment consisted of two experimental blocks for each condition. Individual correct and false responses were recorded. Subjects were given feedback at the end of each block showing their mean RT and the proportion of correct and false responses. Including subject preparation time, the entire experimental session lasted about $4 \mathrm{~h}$.

\section{DATA RECORDING AND ANALYSIS}

EEG activity was recorded with $\mathrm{Ag} / \mathrm{AgCl}$ electrodes from 64 standard locations according to the international 10-20 electrode system using a BIOSEMI Active Two amplifier. Two electrodes specific to the BioSemi acquisition montage (Common Mode Sense and Driven Right Leg) served for reference and ground purpose during the recording. Additional electrodes were placed at the left mastoid (LM) and right mastoid (RM) and at the tip of the nose (serving as offline reference). EOG was measured using the setup described by Schlögl et al. (2007) with one electrode at the nasion and two electrodes below the outer canthi. EEG signals were sampled at $512 \mathrm{~Hz}$. An automatic eye movement correction was applied on the data (Schlögl et al., 2007), preceded by a $0.5-100-\mathrm{Hz}$ offline band-pass filter. After EOG artifact correction, data were filtered offline with a $1-25-\mathrm{Hz}$ band-pass filter. Single trial epochs of $900 \mathrm{~ms}$ duration were extracted containing the 800 -ms following the onset of the sound and additionally a $100-\mathrm{ms}$ pre-stimulus baseline interval, respectively for each condition. Epochs with amplitude changes exceeding $80 \mu \mathrm{V}$ were rejected from further analysis. ERPs were averaged separately for each subject and each condition, with respect to the onset of the sound. Difference waves were formed by subtracting ERPs elicited to the control sound from the ERPs elicited to the corresponding deviant sound for each condition, respectively. For the FM early and FM late condition, MMN was measured at electrode $\mathrm{Fz}$ as the mean amplitude in a $40-\mathrm{ms}$ window around the peak latency of the MMN (Table 1). As under visual inspection no peak in the MMN latency window was identified for the Missing FM early and Missing FM late condition, analogous time windows for the analysis of the mean amplitudes were selected. Presence of MMN was verified by testing the mean amplitude against zero with a one-sample two-tailed Student's $t$-test in each condition. To test for differences in the MMN amplitudes across conditions, a two-way repeated measures analysis of variance (ANOVA) containing the factors Type of deviance (two levels: FM, Missing FM) and Temporal distance (two levels: early, late) was calculated.

Table 1 | Mean MMN amplitudes measured at Fz for the conditions Missing FM early, Missing FM late, FM early, and FM late.

\begin{tabular}{llll}
\hline Condition & Window in ms & Amplitude in $\boldsymbol{\mu V}$ (SD) & $\boldsymbol{t}(\mathbf{d f}=\mathbf{1 2})$ \\
\hline Missing FM early & $238-278$ & $-0.17(0.67)$ & -0.80 n.s. \\
Missing FM late & $511-551$ & $-0.18(1.13)$ & -0.58 n.s. \\
FM early & $238-278$ & $-1.47(1.42)$ & $-3.73^{* *}$ \\
FM late & $511-551$ & $-0.34(0.88)$ & -1.38 n.s.
\end{tabular}

To verify the presence of MMN, one-sample Student's t-tests (see Materials and Methods for details) were applied to test against zero level $\left({ }^{* *} p \leq 0.001\right.$, n.s. non-significant). SD are given in parentheses. (df = degrees of freedom). 
Behavioral data were analyzed with respect to hit rate, false alarm rate, and RTs separately for each subject and each condition. RTs were measured relative to deviance onset. Early $(<100 \mathrm{~ms})$ or late $(>1000 \mathrm{~ms}$ ) responses were rejected from further analysis. Additionally, individual sensitivity indices ( $d^{\prime}$ values) were calculated. In order to avoid infinite $d^{\prime}$ values, 0.5 was added to the number of hits (responses to deviants) and to the number of false alarms (responses to standards), and 1 was added to both the number of deviants and to the number of standards, before calculating hit- and false-alarm rates (Macmillan and Creelman, 1991). By using this adjustment the highest sensitivity a subject could reach by responding 100\% correct and $0 \%$ false would be 5.24. A two-way repeated measures ANOVA with the factors Type of deviance (two levels: FM, Missing FM) and Temporal distance (two levels: early, late) was calculated to compare RTs and $d^{\prime}$ values between conditions, respectively. For EEG- and behavioral-analysis differences between factor levels were tested by calculating pairwise comparisons ( $p$-value alpha-adjusted: $0.0125)$.

\section{RESULTS}

\section{ERP RESULTS}

In Figure 1 grand-average ERP waveforms elicited by deviant sounds and physical identical control sounds as well as the deviant-minus-control difference waves are depicted for all four conditions (Missing FM early, Missing FM late, FM early, and FM late) at the Fz and RM lead. In all conditions sound onsets elicited the P1, N1, and P2 ERP components. Additionally, a sustained potential was observed in ERPs of all conditions. As illustrated in Figure 1 in the FM early condition the deviant-minus-control difference wave shows a negative deflection in the MMN latency range with a polarity inversion at the mastoids. The topography map shows the corresponding distribution for this deflection over the scalp (Figure 2). No such deflections were observed in the MMN latency range for the FM late, Missing FM early, and Missing FM late condition.

The presence of MMN in the FM early condition and the absence of MMN in the other three conditions (FM late, Missing FM early, Missing FM late) were statistically supported by the results of one-sample two-tailed Student's $t$-test (Table 1). Differences in the MMN amplitudes across conditions (FM early, FM late, Missing FM early, and Missing FM late) were verified by a two-way repeated-measurement ANOVA revealing a significant main effect of Type of deviance $[F(1,12)=7.92 ; p=0.016]$. No main effect was obtained for Temporal distance $[F(1,12)=3.31$; $p=0.094]$. Furthermore, a significant interaction of both factors $[F(1,12)=4.23 ; p=0.033]$ was found. Pairwise comparisons indicated that the mean amplitude was significantly more negative in the FM early condition compared to the conditions FM late $[t(12)=-2.53 ; p=0.026]$ and Missing FM early $[t(12)=-3.10$;
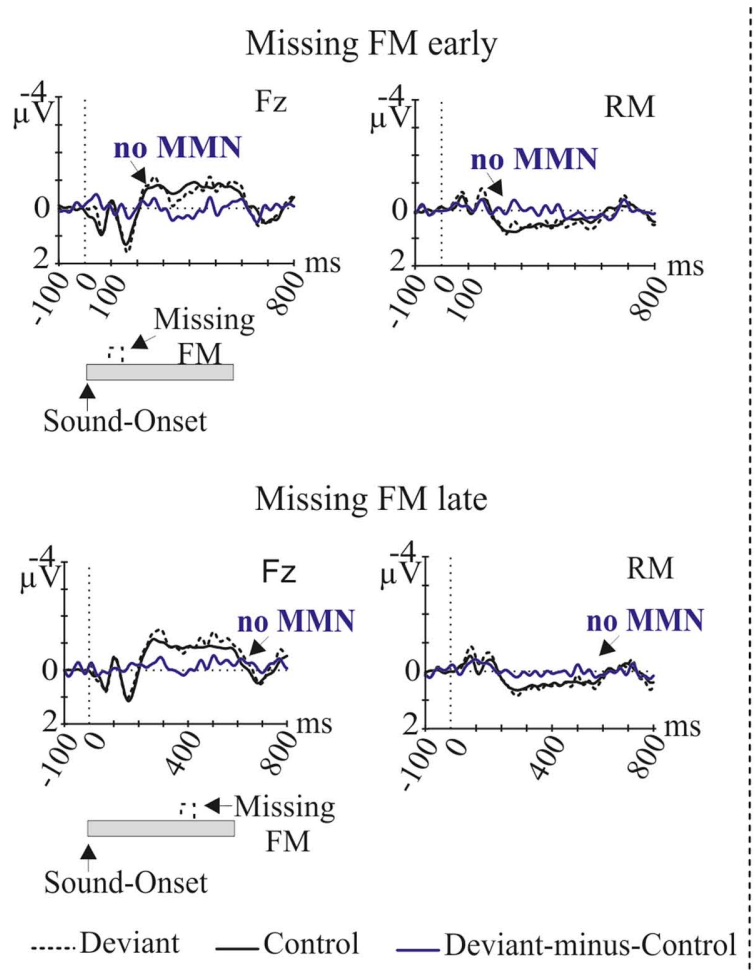

FIGURE 1 | Grand-average ERP waves (at Fz and RM), elicited by deviant sounds (black dotted line) and by physical identical control sounds (black solid line), separately for the conditions Missing FM early, Missing FM late, FM early, and $F \mathbf{M}$ late. The corresponding deviant-minus-control difference wave is depicted

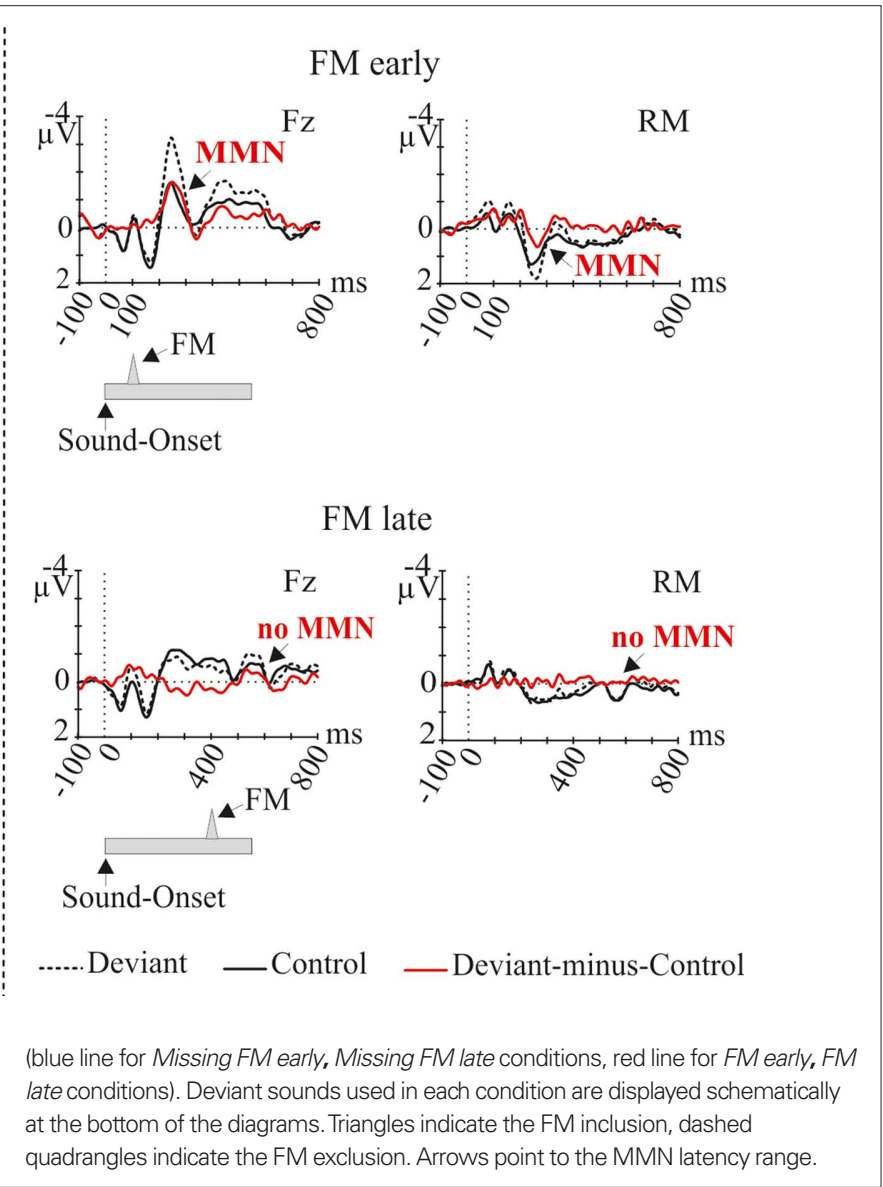


$p=0.009]$. No differences in MMN amplitudes were obtained comparing FM late to Missing FM late condition $[t(12)=-0.58$; $p=0.571]$ and Missing FM early to Missing FM late condition $[t(12)=0.06 ; p=0.954]$.

\section{BEHAVIORAL RESULTS}

Table 2 summarizes the behavioral results (hit rates, false alarm rates, RTs, and $d^{\prime}$ values) obtained in response to the deviants. In all conditions deviants were detected with high accuracy. However, repeated measures ANOVA comparing $d^{\prime}$ values across conditions revealed significant main effects of Type of deviance $[F(1,12)=10.93 ; p=0.006]$ and Temporal distance $[F(1,12)=7.78$; $p=0.016]$ as well as a significant interaction of both factors $[F(1,12)=7.94 ; p=0.016]$. Pairwise comparison between the four experimental conditions revealed that the sensitivity was significantly higher for the condition FM late than for Missing FM late $[t(12)=4.58 ; p=0.001]$. Similarly, in the condition Missing FM early sensitivity was higher than in the condition Missing FM late $[t(12)=3.55 ; p=0.004]$, whereas sensitivity between conditions FM early and Missing FM early $[t(12)=1.52 ; p=0.155]$ as well as FM early and FM late $[t(12)=0.23 ; p=0.820]$ did not differ (Figure 3).

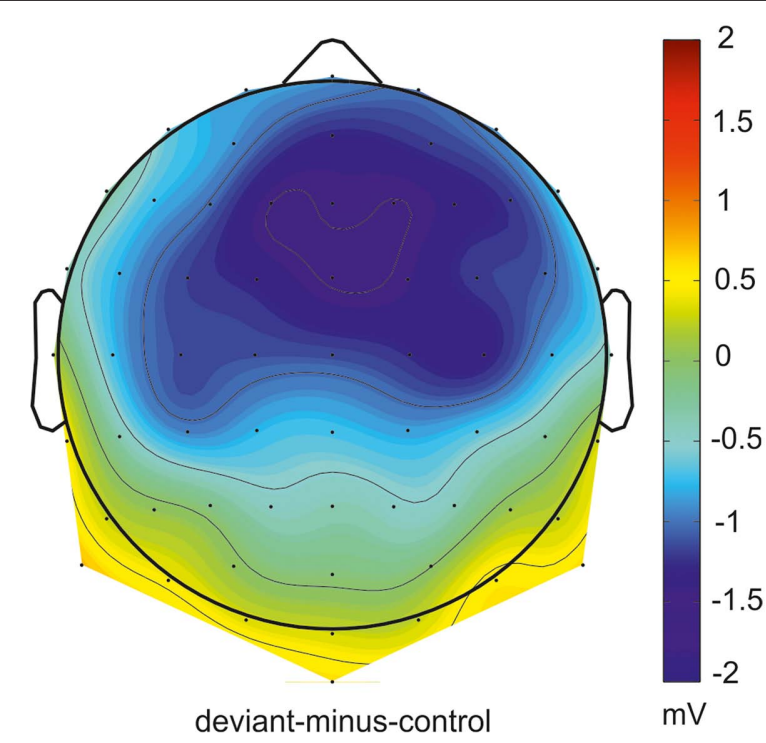

FIGURE 2 |Topographical distribution of absolute MIMN voltages for the FM early condition in the time window from 238 to $278 \mathrm{~ms}$ relative to sound onset.
With respect to the comparison of RTs across conditions repeated measures ANOVA showed a significant main effect of Type of deviance $[F(1,12)=13.75 ; p=0.003]$. No main effect was obtained for Temporal distance $[F(1,12)=0.01 ; p=0.947]$. Furthermore, no interaction was found $[F(1,12)=0.63 ; p=0.445]$. The main effect in Type of deviance resulted from significant faster RTs in the FM conditions than in the Missing FM conditions (Figure 4).

\section{DISCUSSION}

The present study tested to what extent a rare deviant sound, which does not vary in spectral content over time, is discriminated by the deviance detection system when occurring among regular time-variant sounds. For this purpose we measured MMN to the infrequent exclusion of a transient FM that formed part of regular occurring pure sine wave tones at different temporal positions (Missing FM early, Missing

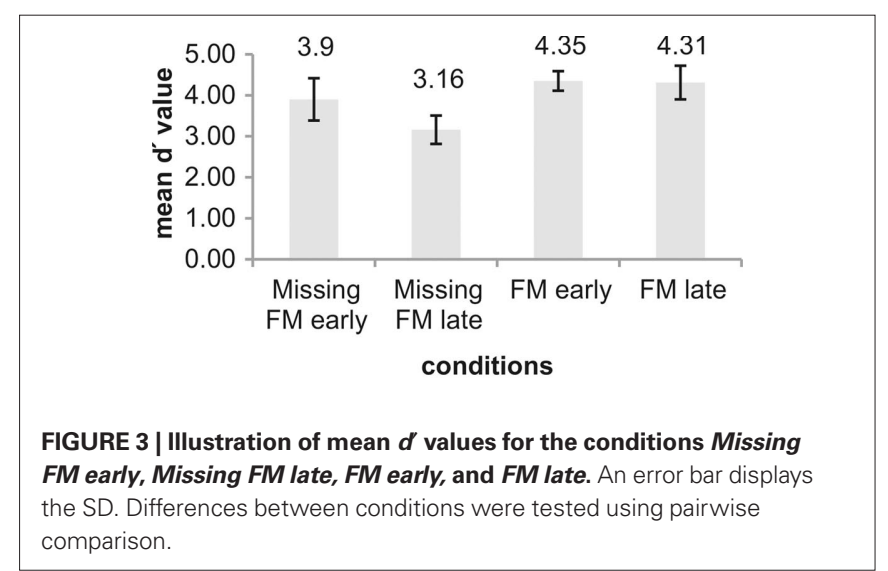

\begin{tabular}{|c|c|c|c|c|c|}
\hline 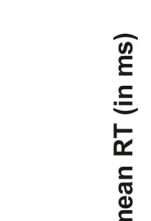 & $\begin{array}{l}600 \\
500 \\
400 \\
300 \\
200 \\
100\end{array}$ & $\begin{array}{c}512 \\
I\end{array}$ & $\begin{array}{c}518 \\
I\end{array}$ & $\begin{array}{c}446 \\
I\end{array}$ & $\begin{array}{c}442 \\
I\end{array}$ \\
\hline & & $\begin{array}{l}\text { Missing } \\
\text { FM early }\end{array}$ & $\begin{array}{r}\text { Missing } \\
\text { FM late } \\
\text { con }\end{array}$ & $\begin{array}{l}\text { FM early } \\
\text { ditions }\end{array}$ & FM late \\
\hline $\begin{array}{l}\text { FIGURE } 4 \text { | III } \\
\text { FM early, Mis } \\
\text { SD. Difference } \\
\text { comparisons. }\end{array}$ & $\begin{array}{l}\text { ustrat } \\
\text { sing } \boldsymbol{F} \\
\text { s betv }\end{array}$ & $\begin{array}{l}\text { ion of mean } \\
\text { M late, } \mathbf{F M} \\
\text { veen conditi }\end{array}$ & $\begin{array}{l}\text { RTs (in } \mathrm{m} \\
\text { early, and } \\
\text { ons were t }\end{array}$ & $\begin{array}{l}\text { s) for the cc } \\
\text { FM late. An } \\
\text { ested using }\end{array}$ & $\begin{array}{l}\text { nditions Missing } \\
\text { error bar displays the } \\
\text { Dairwise }\end{array}$ \\
\hline
\end{tabular}

Table 2 | Behavioral data obtained in the deviant detection task for the conditions Missing FM early, Missing FM late, FM early, and FM late.

\begin{tabular}{|c|c|c|c|c|}
\hline Condition & Hits in \% (SD) & False alarms in \% (SD) & Reaction times in ms (SD) & $d^{\prime}$ values (SD) \\
\hline Missing FM early & $90.33(12.34)$ & $1.50(1.58)$ & $512(84)$ & $3.90(1.03)$ \\
\hline Missing FM late & $78.52(11.29)$ & $1.67(1.93)$ & $518(84)$ & $3.16(0.69)$ \\
\hline FM early & $92.03(7.59)$ & $0.42(0.44)$ & $446(82)$ & $4.35(0.48)$ \\
\hline FM late & 91.65 (11.46) & $0.59(0.49)$ & $442(79)$ & $4.31(0.82)$ \\
\hline
\end{tabular}

SD are given in parentheses. 
FM late condition). For comparison purposes we measured MMN also in the reversed case, that is, to the infrequent inclusion of a transient FM at different temporal positions that was absent in the regularly occurring pure sine wave tones (FM early, FM late condition).

\section{AN ASYMMETRY IN THE AUTOMATIC DETECTION OF A DEVIANT INCLUSION OR EXCLUSION OF FM}

We found MMN only when the deviant featured an early occurring transient FM (FM early condition) with a typical MMN scalp distribution (as previously shown by Grimm et al., 2006). No MMN was elicited in all the other conditions (FM late, Missing FM early, Missing FM late). This result shows that it makes a difference for the automatic deviance detection system whether the deviance consists in the irregular inclusion or the irregular exclusion of a transient stimulus feature. Thus, the current data support the feature-detector hypothesis as the feature detectors should be activated to a larger extent when the deviance is defined by the inclusion of a transient feature (Nordby et al., 1994; Sabri and Campbell, 2000; Bishop et al., 2005).

However, as known from previous studies, a rare feature exclusion can elicit an MMN (Nordby et al., 1994; Sabri and Campbell, 2000). Thus, we did not expect the total absence of MMN to the exclusion of the early transient FM. Sabri and Campbell (2000) found an MMN-like component of attenuated size when the deviance was the exclusion of a salient click that formed part of frequently presented white noise. Also, Nordby et al. (1994) found an attenuated MMN when the deviance typified a feature exclusion (deviant: white noise without $1000-\mathrm{Hz}$; standard: white noise with $1000-\mathrm{Hz}$ ). Both studies found a MMN of larger size when the deviant featured the inclusion and the standard the exclusion of the additional feature. Indeed, a paper by Bishop et al. (2005) reported a similar pattern of results, although the deviating feature was not transient but affected the whole sound in their study. Bishop et al. (2005) found no MMN when deviants were sounds of constant frequency occurring in the context of one of several different spectro-temporally modulated standard sounds (perceived like a trill, a wobble or a chord). On the other hand, when the standard was a pure tone and the deviant sound was one of the modulated sounds, they found MMN. However, they did not provide additional behavioral information that might help to understand the total absence of MMN for the exclusion. Taken together, it seems that auditory stimuli lacking any spectral variation do not elicit MMN when occurring in a context of standard stimuli that contain a variation either affecting the whole sound as in Bishop et al. (2005) or a particular portion of the sound as shown in the present study. It can be speculated that the time-variant structure of white noise deviants used in other studies (Nordby et al., 1994; Sabri and Campbell, 2000) can account for the presence of MMN (though of smaller amplitude) they found in response to the exclusion of an additional feature.

\section{BEHAVIORAL DETECTION OF A DEVIANT INCLUSION OR EXCLUSION OF FM}

In the current behavioral task, listeners' detection performance of the early FM was of comparable accuracy irrespective of whether the deviance characterized the inclusion $\left(d^{\prime}=4.35\right)$ or exclusion $\left(d^{\prime}=3.9\right)$. Thus, the lack of MMN for the exclusion is not accompanied by a weaker discrimination performance to this kind of deviance per se. On the contrary, for the late FM a clear difference between inclusion deviants $\left(d^{\prime}=4.31\right)$ and exclusion deviants $\left(d^{\prime}=3.16\right)$ was observed. This pattern of results is partly in line with Sabri and Campbell (2000) who also reported a much better detection performance for the inclusion of a click in the deviant sound than for its exclusion for the less salient clicks. Further, several behavioral studies observed a similar perceptual asymmetry, obtaining lower sensitivity indices for the feature exclusion compared to its inclusion in the deviant sound (Asemi et al., 2003; Cusack and Carlyon, 2003).

Sensitivity indices did not differ between early and late occurrence for the FM deviant. However, when the deviance was the exclusion of the FM, we found on behavioral level for the early exclusion a better detection performance with respect to sensitivity indices (even though not for RTs) than that for the late one. This diverging pattern could be explained by a ceiling effect that is present for the FM deviant sound, which might not be present when the FM is omitted from the deviant sound.

Apparently, the pattern of behavioral data does not correspond to the pattern of MMN reflecting the accuracy of the automatic sound representation. Whereas deviants containing a late FM do not elicit an MMN, behavioral performance is comparable to deviants that contain an early FM. That is, under attention discriminating the deviant from the standard sounds does not necessarily depend on the function of automatic deviance detection.

An interaction more difficult to explain is the absence of MMN for the early exclusion deviant, whereas detection accuracy was comparable to that of the early FM deviant, which furthermore elicited MMN. Again, active detection performance appears to not solely rely on the output of the automatic deviance detection system, but cannot be based on low-level feature detection either, as the deviant does not contain the differentiating transient feature. That is, under attention the system is capable of discriminating exclusion deviants on the basis of the mere difference in the informational content between standard and deviant, irrespective whether the additional feature is in the standard or in the deviant.

Moreover, the present study rather suggests a time-precise than a holistic processing for the feature exclusion in the deviant sound. That is, not the holistic organization but rather the spectro-temporal sound representations are compared along the time axis to perform the discrimination (as already suggested by Grimm and Schröger, 2007). RTs were in general slower for feature exclusions, but there was no interaction of deviant type and time of occurrence. That is, for both exclusions and inclusions, attentive deviance detection is time-locked to the (potential) point of occurrence of the relevant transient feature, even though in the case of exclusions the system - once triggered - might need more time to accumulate enough evidence in order to make a decision.

\section{THE TEMPORAL DISTANCE EFFECT}

As expected, we found the temporal distance effect (Näätänen et al., 2004; Grimm and Schröger, 2005; Grimm et al., 2006; Weise et al., 2010) on MMN amplitude when the deviant contained a FM early vs. late following sound onset. That is, an MMN was elicited to an early FM whereas no MMN was elicited to a late FM in the deviant sound. However, as we did not obtain MMN when the deviant was 
characterized by the exclusion of an early or late FM, we cannot make inferences on whether a temporal distance effect does or does not exist in such a case.

Concerning the behavioral data, we did not expect a temporal distance effect on sensitivity indices and RTs for rare feature inclusions and exclusions as a previous study demonstrated that with focused attention no such effect occurs on the level of detection performance (Grimm, 2009). This assumption was confirmed by the results obtained to deviants that featured an early vs. late FM: RTs and sensitivity indices did not differ. When the deviance was the exclusion of the FM, we unexpectedly found on the behavioral level better detection performance with respect to sensitivity indices (even though not for RTs) for the early exclusion than that for the late one. It is possible that when the FM is part of the deviant sound there might be a ceiling effect, whereas this might not be the case when the FM is omitted from the deviant sound. Another possible explanation might be the temporal uncertainty when the omission of the FM occurs. This uncertainty in the temporal occurrence might be more pronounced the later the deviance occurs.

\section{INTERPRETATION OF THE RESULTS WITHIN A LARGER FRAMEWORK CONCERNING THE CURRENT ACCOUNTS OF MMN}

The mechanism underlying the elicitation of MMN is usually described as a comparison of auditory sensory memory representations of the predictive regularity and the unpredicted deviant (e.g., Näätänen, 1990, 1992; Näätänen et al., 2005). According to an alternative, (almost) as powerful account MMN elicitation is due to activation of fresh-afferent cortical neurons responding to the deviant, thereby overcoming the neural adaptation to the standards (e.g., Ullsperger and Baldeweg, 1990; Jääskeläinen et al., 2004; May and Tiitinen, 2010). The difference between these two accounts partly seems to result from a difference in the conceptual level dealing with MMN. The memory-comparison based account is merely formulated at a cognitive-psychological level, whereas the fresh-afferent neuronal activity account is merely formulated at a neurophysiological level. However, despite this difference at the conceptual level utilized to explain the MMN, there seems to exist a core difference in the two accounts: Whereas the memory-comparison based account postulates that the $\mathrm{MMN}$ is due to additional neural activation when an irregular event is detected (Näätänen et al., 2011) similar to other prediction-error related signals such as the error-negativity (e.g., Falkenstein et al., 2000) - the neural adaptation account postulates that MMN is not the result of an additional, genuine process but rather an enhanced N1 (May and Tiitinen, 2010).

Obviously, the additional-feature-detector hypothesis (Bishop et al., 2005), which is suited to explain the absence of MMN to the rare exclusion of a FM observed in the present study, forms a special case of the fresh-afferent neuronal activity account for MMN (e.g., May and Tiitinen, 2010). Thus, following the law of parsimony, our results seem to be in favor of the fresh-afferent neuronal activity account of MMN. However, this does - of course - not rule out the existence of the memory-based comparison mechanism. But how can we then explain the absence of MMN to the exclusion of a FM in the present study? In our opinion, it seems possible that the postulated memory-based comparison mechanism relies on an exact temporal match between the regularity representation and the current sound. As the deviant did not include a transient (except sound onset) the comparison mechanism may have not been able to temporarily align the deviant information to the regularity representation. In other words, the comparison mechanism seems to need a transient in order to being able to work properly. As such transients result in the elicitation of $\mathrm{N} 1$, it is suggested that the processes underlying $\mathrm{N} 1$ may play an important role in aligning the representations of deviant and standard underlying MMN. The functional significance of $\mathrm{N} 1$ as an indicator for segmenting the sound input (Fishbach et al., 2001), feature encoding (Näätänen, et al., 2011), and initializing new regularities (Winkler et al., 2009) has already been acknowledged in previous studies. The present suggestion, according to which the processes underlying $\mathrm{N} 1$ also serve the correct temporal alignment of the comparison process underlying $\mathrm{MMN}$, is rather speculative. However, there exists corroborating evidence for this idea from structurally similar MMN findings: Although MMN elicited by intensity decrements increases with decreasing the intensity of the deviant over a wide intensity range (e.g., Näätänen et al., 1989), no $\mathrm{MMN}$ is elicited when the deviant becomes too soft or when the sound is omitted (e.g., Yabe et al., 1997; Takegata et al., 2011; unless the omission falls within the temporal window of integration). Again, in the absence of a proper perceptual unit with a fixed onset, the comparison mechanism can hardly align the "deviant" information to the regularity representation. Importantly, also the fresh-afferent neuronal activity account of MMN cannot explain why there is no MMN in the FM late condition, although there is a transient in the sound which is sufficient to result in MMN in the FM early condition. One might wonder, whether the lack of MMN in FM late is due to a jitter in the deviance detection process. However, this can be ruled out as in the individual ERPs from 12 of 13 participants no "smeared" MMN is observable. Thus, neither the fresh-afferent neuronal activity nor the memory-based comparison account of MMN can satisfactorily explain the full pattern of results.

\section{CONCLUSION}

The present study revealed that the automatic deviance detection system is most sensitive to a deviant sound that includes an additional, early occurring feature (pure tone with brief FM) that is absent in the standard sound (pure tone). On the other hand, the deviance detection system is less sensitive (or even insensitive) to a deviant sound that excludes an additional feature (pure tone) that is present in the standard sound (pure tone with brief FM). Even though from a mere informational point of view, the relative magnitude of the deviance is comparable in both cases (which is also indicated by comparable $d^{\prime}$ values for detecting the absence vs. presence of the FM in the deviant), the automatic deviant detection seems not to use this information. This asymmetry in automatic deviant detection rather relies on the increased activity of feature detectors to additional features within sounds supporting the assumption of the feature-detector hypothesis (Nordby et al., 1994; Sabri and Campbell, 2000; Bishop et al., 2005). If the fresh-afferent neuronal activity account and the memory-based comparison account of MMN were really mutually exclusive (as sometimes stated in the literature), the present results would be more parsimoniously explained with the former. However, the absence of MMN to the exclusion of a feature can also be explained with the memory-based comparison account as the comparison 
can hardly take place without a temporarily precise timing, which is not possible when information about the onset of the deviance is not provided (as it is the case with FM exclusions).

\section{ACKNOWLEDGMENTS}

This work was supported by the German Research Foundation (Deutsche Forschungsgemeinschaft, DFG, Project 375/19-1), by the German Academic Exchange Service (Deutscher Akademischer

\section{REFERENCES}

Asemi, N., Sugita, Y., and Suzuki, Y.(2003). Auditory search asymmetry between pure tone and temporal fluctuating sounds distributed on the frontalhorizontal plane. Acta Acustica 89, 346-354.

Bishop,D.V.M., O’Reilly, J., and McArthur, G.M. (2005). Electrophysiological evidence implicates automatic low-level feature detectors in perceptual asymmetry. Cogn. Brain Res. 24, 177-179.

Cusack, R., and Carlyon, R. P. (2003). Perceptual asymmetries in audition. J. Exp. Psychol. Hum. 29, 713-725.

Falkenstein, M., Hoormann, J., Christ, S., and Hohnsbein, J. (2000). ERP components on reaction errors and their functional significance: a tutorial. Biol. Psychol. 51, 87-107.

Fishbach, A., Nelken, I., and Yeshurun, Y. (2001). Auditory edge detection: a neural model for physiological and psychoacoustical responses to amplitude transients. J. Neurophysiol. 85, 2303-2323.

Grimm, S. (2009). Representations of Auditory Time: Pre-Attentive and Attentive Processing of Temporal Sound Features. Leipzig: Leipziger Universitätsverlag.

Grimm, S., Röber, U., Trujillo-Barreto, N., and Schröger, E. (2006). Mechanisms for detecting auditory temporal and spectral deviations operate over similar time windows but are not divided differently between the two hemispheres. Neuroimage 32, 275-282.

Grimm, S., and Schröger, E. (2005). Preattentive and attentive processing of temporal and frequency characteristics within long sounds. Cogn. Brain Res. 25, 711-721.

Grimm, S., and Schröger, E. (2007). The processing of frequency deviations within sounds: evidence for the predictive nature of the mismatch negativity (MMN) system. Restor. Neurol. Neurosci. 25, 241-249.

Jääskeläinen, I. P., Ahveninen, J., Bonmassar, G., Dale, A. M., Ilmoniemi, R. J., Levänen, S., Lin, F. H., May, P., Melcher, J., Stufflebeam, S., Tiitinen, H., and Belliveau, J.W. (2004). Human posterior auditory cortex gates novel sounds to consciousness. Proc. Natl. Acad. Sci. U.S.A. 101, 6809-6814.

Kaernbach, C. (1990). A single-interval adjustment-matrix (SIAM) procedure for unbiased adaptive testing. J. Acoust. Soc. Am. 88, 2645-2655.

Kujala, T., Tervaniemi, M., and Schröger, E. (2007). The mismatch negativity in cognitive and clinical neuroscience: theoretical and methodological considerations. Biol. Psychol. 74, 1-19.

Macmillan, N. A., and Creelman, C. D. (1991). Detection Theory: A User's Guide. New York: Cambridge University Press.

May, P. J. C., and Tiitinen, H. (2010). Mismatch negativity (MMN), the deviance-elicited auditory deflection, explained. Psychophysiology 46, 66-122.

Näätänen, R. (1990). The role of attention in auditory information processing as revealed by event-related potentials and other brain measures of cognitive function. Behav. Brain Sci. 13, 201-288.

Näätänen, R. (1992). Attention and Brain Function. Hillsdale, NJ: Lawrence Erlbaum Associates.

Näätänen, R., Gaillard, A. W. K., and Mäntysalo, S. (1978). Early selectiveattention effect on evoked-potential reinterpreted. Acta Psychol. 42, 313-329.

Näätänen, R., Jacobsen, T., and Winkler, I. (2005). Memory-based or afferent processes in mismatch negativity (MMN): a review of the evidence. Psychophysiology 42, 25-32.

Näätänen, R., Kujala, T., and Winkler, I. (2011). Auditory processing that leads to consciousperception: a unique window to central auditory processing opened by the mismatchnegativity and related responses. Psychophysiology 48, 4-22.

Näätänen, R., Paavilainen, P., Alho, K., Reinikainen, K., and Sams, M. (1989). Do event-related potentials reveal the mechanism of the auditory sensory memory in the human brain? Neurosci. Lett. 98, 217-221.

Näätänen, R., Paavilainen, P., Rinne, T., and Alho, K. (2007). The Mismatch

Austauschdienst, DAAD, Project 0811948), and by the Spanish ministry of Science and Innovation (Acciones Integradas grant HD 2008-10). It was realized by using Cogent 2000 developed by the Cogent 2000 team at the FIL and the ICN (http://www.vislab.ucl. ac.uk). The authors wish to thank Andreas Widmann for providing the scripts for eye movement correction and hearing threshold adjustment and Martin Reiche and Anne Steiger for assistance in data acquisition.

negativity (MMN) in basic research of central auditory processing: a review. Clin. Neurophysiol. 118, 2544-2590.

Näätänen, R., Sysoeva, O., and Takegata, R. (2004). Automatic time perception in the human brain for intervals ranging from milliseconds to seconds. Psychophysiology 41, 660-663.

Nordby, H., Hammerborg, D., Roth, W. T., and Hugdahl, K. (1994). ERPs for infrequent omissions and inclusions of stimulus elements. Psychophysiology 31, 544-552.

Sabri, M., and Campbell, K. B. (2000). Mismatch negativity to inclusions and omissions of stimulus features. Neuroreport 11, 1503-1507.

Schlögl,A., Keinrath, C., Zimmermann, D., Scherer, R., Leeb, R., and Pfurtscheller, G. (2007). A fully automated correction method of EOG artifacts in EEG recordings. Clin. Psychol. 118, 98-104.

Sinkkonen, J. (1999). "Information and resource allocation," in Information Theory and the Brain, eds $\mathrm{R}$. Baddeley, P. Hancock, and P. Foldiak, (Cambridge: Cambridge University Press), 241-254.

Sinkkonen, J., Kaski, S., Huotilainen, M., Ilmoniemi, R., Näätänen, R., and Kaila, K. (1996). Optimal resource allocation for novelty detection in a human auditory memory.Neuroreport 7,2479-2482.

Takegata, R., Heikkilä, R., and Näätänen, R. (2011). Sound energy and the magnitude of change: effects on mismatch negativity. Neuroreport 22, 171-174.

Ullsperger, P., and Baldeweg, T. (1990) Sensory adaptation and mismatch negativity. Behav. Brain Sci.13, 255-255.

Warren, R. M. (1993). "Perception of acoustic sequences: global integration versus temporal resolution," in Thinking in Sounds: The Cognitive Psychology of Human Audition, eds S. McAdams and E. Bigand (Oxford: Oxford University Press), 37-68.

Warren, R. M., and Ackroff, J. M. (1976). Two types of auditory sequence perception. Percept. Psychophys. 20, 387-394.

Weise, A., Grimm, S., Müller, D., and Schröger, E. (2010). A temporal constraint for automatic deviant detection and object formation: a mismatch negativity study. Brain Res. 1331, 88-95.

Weise, A., Müller, D., Grimm, S., Rübsamen, R., and Schröger, E. (2007). Differential processing of terminal tone parts within structured and non-structured tones. Neurosci. Lett. 421, 163-167.

Winkler, I., Denham, S. L., and Nelken, I. (2009). Modeling the auditory scene: predictive regularity representations and perceptual objects. Trends Cogn Sci. (Regul. Ed.) 13, 532-540.

Winkler, I., and Näätänen, R. (1993). "Event-related brain potentials to infrequent partial omissions in series of auditory stimuli," in Perspectives in Cognitive Neurophysiology, eds $\mathrm{H}$. -J. Heinze, G. R. Mangun, and T. F. Münte (Boston, MA: Birkhäuser), 219-226.

Yabe, H., Tervaniemi, M., Reinikainen, K., and Näätänen, R. (1997). Temporal window of integration revealed by MMN to sound omission. Neuroreport 8, 1971-1974.

Conflict of Interest Statement: The authors declare that the research was conducted in the absence of any commercial or financial relationships that could be construed as a potential conflict of interest.

Received: 29 April 2011; accepted: 25 July 2011; published online: 08 August 2011.

Citation: Timm J, Weise A, Grimm $S$ and Schröger E (2011) An asymmetry in the automatic detection of the presence or absence of a frequency modulation within a tone: a mismatch negativity study. Front. Psychology. 2:189. doi: 10.3389/ fpsyg.2011.00189

This article was submitted to Frontiers in Auditory Cognitive Neuroscience, a specialty of Frontiers in Psychology.

Copyright (c) 2011 Timm, Weise, Grimm and Schröger. This is an open-access article subject to a non-exclusive license between the authors and Frontiers Media SA, which permits use, distribution and reproduction in other forums, provided the original authors and source are credited and other Frontiers conditions are complied with 